\title{
OPEN Ultrasound probe tilt impedes the needle-beam alignment during the ultrasound-guided procedures
}

\author{
Qingxiang $\mathrm{MaO}^{1}$, Haitao $\mathrm{He}^{2}$, Yuangang $\mathrm{Lu}^{3}$, $\mathrm{Yi} \mathrm{Hu}^{1}$, Zhen Wang ${ }^{1}$, Maoxiang Gan ${ }^{1}$, \\ Hong $\operatorname{Yan}^{1 \bowtie}$ \& Liyong Chen ${ }^{1 \bowtie}$
}

The objective of this study was to identify the factors that complicate the needle visualization in ultrasound-guided in-plane needling procedures. Forty-nine residents were recruited and randomized to insert the simulated blood vessel with four different views including Neutral (the long axis of the probe along the visual axis and the ultrasonic beam vertical to the surface of gel phantom), $45^{\circ}$-rotation ( $45^{\circ}$ angle between the long axis of probe and the operator's visual axis), $45^{\circ}$-tilt $\left(45^{\circ}\right.$ angle between the ultrasonic beam and the surface of gel phantom) and $45^{\circ}$-rotation plus $45^{\circ}$-tilt of probe. Number of needle redirections, insertion time, and needle visibility were documented and compared for each procedure. When the residents faced with $45^{\circ}$-tilt view, the needle redirections ( 2 vs 0 ) and insertion time increased significantly ( 39 vs 16 ) compared with that of the Neutral view. When faced with $45^{\circ}$-rotation plus $45^{\circ}$-tilt view, the residents' performance decreased further as compared with that of the $45^{\circ}$-tilt view and the Neutral view. However, there was no performance difference between the Neutral view and $45^{\circ}$-rotation view. In conclusion, during ultrasoundguided in-plane procedures, tilting the ultrasound probe may increase the difficulty of needle-beam alignment.

In ultrasound-guided regional anesthesia, the in-plane technique allows precise targeting of the nerve and realtime visualization of needle trajectory ${ }^{1,2}$. Nevertheless, failure to align needle and ultrasonic beam imposes the risk of accidentally damaging adjacent vital structures not visible on the ultrasound screen ${ }^{3,4}$. Tilting and rotating are common movements of probe and they help to optimize the visualization of the targeted nerves or vessels. During the ultrasound-guided supraclavicular brachial plexus block, for example, the transducer is always placed in an oblique transverse plane (rotation) and tilted to supraclavicular fossa (tilt) ${ }^{5,6}$. However whether these maneuvers themselves have any potential influences on needle-beam alignment is not known.

In the present study, we compared the resident volunteers' performance of ultrasound-guided vessel insertion in four different standard scenarios including Neutral, $45^{\circ}$-rotation, $45^{\circ}$-tilt and $45^{\circ}$-rotation plus $45^{\circ}$-tilt of probe. We hypothesized that these manipulation maneuvers would increase the difficulty of needle-beam alignment and decrease the success rates.

\section{Methods}

The study protocol was adhered to the Declaration of Helsinki and was approved by the Ethics Committee of the Daping Hospital (2018-45). Forty-nine Anesthesia residents were enrolled in the phantom study and the informed consent was obtained from all subjects. Before tasks, participants were asked to complete a questionnaire about experiences in ultrasound-guided procedure. For residents who have no experience were provided with a 10-min standardized ultrasound-guided in-plane insertion instruction. Participants were asked to insert the long-axis of simulated blood vessel on a gel phantom (Ningbo Liuyedao Medical Technology Co., Ltd, China) with four different views including Neutral (the long axis of the probe was along the visual axis and the ultrasonic beam was vertical to the surface of gel phantom), $45^{\circ}$-rotation (there was $45^{\circ}$ angle between the long axis of probe

${ }^{1}$ Department of Anesthesiology, Daping Hospital, Army Medical University, 10 ChangjiangZhilu, Yuzhong District, Chongqing 400042, China. 'Department of Maxillofacial and Head and Neck Surgery, Daping Hospital, Army Medical University, Chongqing, China. ${ }^{3}$ Department of Plastic and Cosmetic Surgery, Daping Hospital, Army Medical University, Chongqing, China.『email: cqyanhong@163.com; mzkcly@aliyun.com 

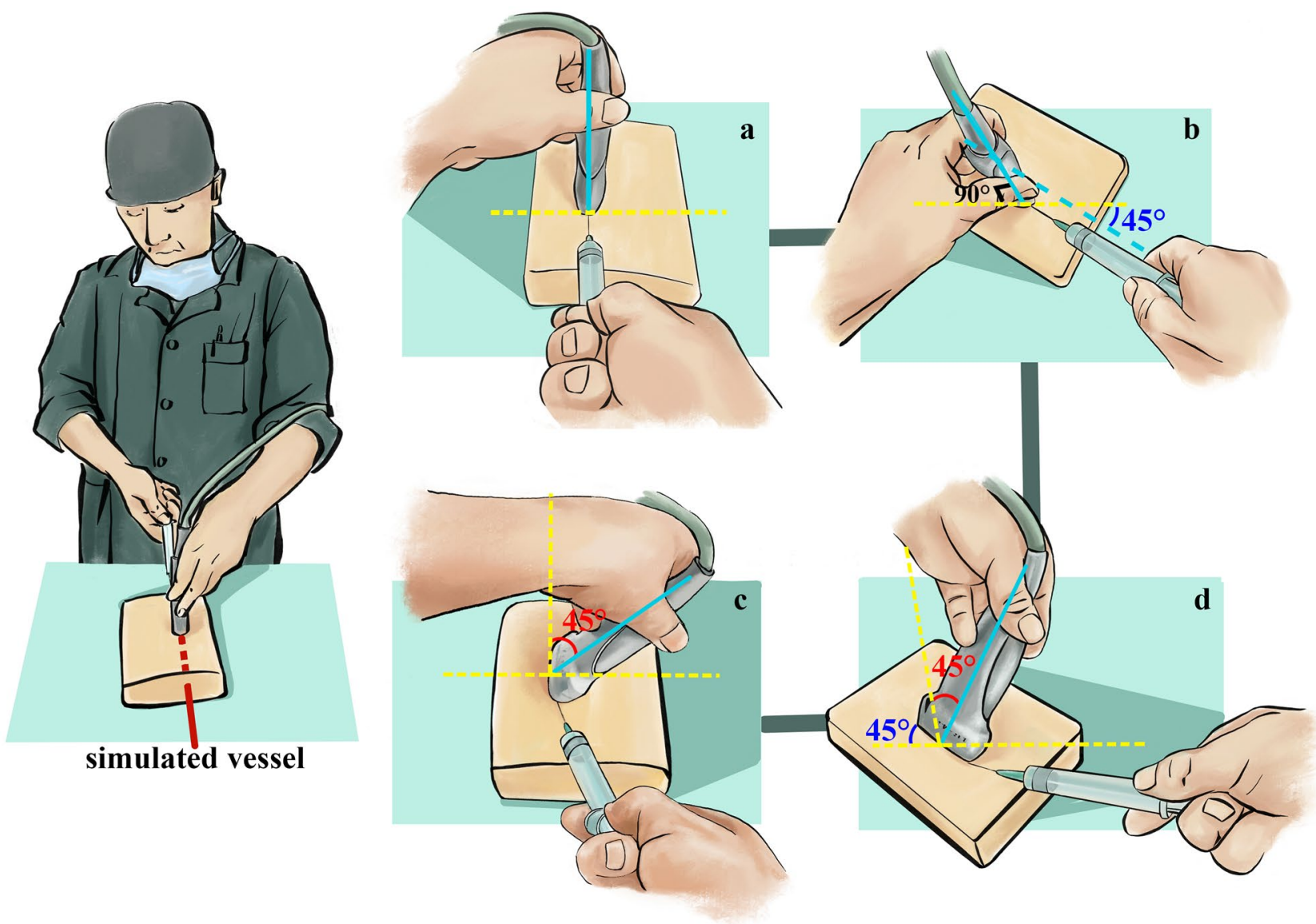

Figure 1. A schematic drawing of the four insertion views in phantom study. The simulated vessel is represented by the red dashed line. (a) Neutral view, the long axis of ultrasound probe was along the operator's visual axis and ultrasonic beam was vertical to the surface of gel phantom; (b) $45^{\circ}$-rotation view, there is a $45^{\circ}$ angle between the long axis of probe and the operator's visual axis (or sagittal plane); (c) $45^{\circ}$-tilt view, there is a $45^{\circ}$ angle between the ultrasonic beam and the vertical line (or the surface of gel phantom); (d) $45^{\circ}$-rotation plus $45^{\circ}$-tilt view, there is $45^{\circ}$ angle between the long axis of probe and the operator's visual axis (or sagittal plane), while there is another $45^{\circ}$ angle between the ultrasonic beam and the vertical line. Yellow dashed line: Vertical/ horizontal reference line; Blue solid line: direction of ultrasonic beam; Green angle: rotation angle; Red angle: tilt angle. The copyright holder of this image has been acknowledged and agreed to publish it under a CC BY open access license.

and the operator's visual axis), $45^{\circ}$-tilt (there was $45^{\circ}$ angle between the ultrasonic beam and the surface of gel phantom) and $45^{\circ}$-rotation plus $45^{\circ}$-tilt of probe (Fig. 1a-d). A M9 ultrasound machine (Mindray, Shenzhen, China) equipped with a high-resolution $9.0-\mathrm{MHz}$ linear probe was used. The order in which insertion was performed with each view was determined by a computer-generated random number list. Successful insertion of vessel was defined as aspiration of fluid from the simulated vessel. All attempts were recorded by a video camera and analyzed by an attending physician. After completion of the tasks, all participants were asked to rank the four views from easiest to the most difficult. The participants and analyst were blinded to the study objectives.

The performance outcome data are reported as medians with interquartile ranges (IQRs). Statistical analysis was conducted by Mann-Whitney U test or Friedman's two-way test with post hoc analysis using Dunn-Bonferroni test to compare the performance outcome. Demographic characteristics of study participants are reported as mean \pm SD. Statistical analysis was conducted by one-way ANOVA or chi-squared. The level of significance was set at $P<0.05$.

\section{Results}

When faced with Neutral view (Fig. 1a), the operators showed the best performance outcome (Table 1). When there was $45^{\circ}$ angle between the long axis of probe and the operator's visual axis (rotation), the operator showed similar performance outcome with that in Neutral view.

When faced with $45^{\circ}$-tilt view, the number of needle redirections $(2$ vs $0, P=0.012 ; 2$ vs $0, P=0.037)$ and insertion time ( 39 vs $16, P=0.003 ; 39$ vs $20, P=0.003$ ) increased significantly as compared with the Neutral and the $45^{\circ}$-rotation group.

When faced with the $45^{\circ}$-rotation plus $45^{\circ}$-tilt view, the operators unsurprisingly showed the worst performance outcome (Table 1). The number of skin breaks, needle redirections and the insertion time increased 


\begin{tabular}{|c|c|c|c|c|}
\hline Variables & Neutral & $45^{\circ}$ rotation & $45^{\circ}$ tilt & $45^{\circ}$ rotation and $45^{\circ}$ tilt \\
\hline \multicolumn{5}{|c|}{ Skin breaks (times) } \\
\hline All residents & $1(1-1)$ & $1(1-1)$ & $1(1-2)$ & $3(1-6)^{a, b}$ \\
\hline Level $1(n=15)$ & $1(1-2)$ & $1(1-2)$ & $1(1-2)$ & $4(1-6.5)$ \\
\hline Level $2(\mathrm{n}=16)$ & $1(1-1)$ & $1(1-1)$ & $1(1-3.3)$ & $3(1-6.3)$ \\
\hline Level $3(n=18)$ & $1(1-1)^{\mathrm{c}}$ & $1(1-1)$ & $1(1-2.3)$ & $2(1-3.4)$ \\
\hline \multicolumn{5}{|c|}{ Needle redirections (times) } \\
\hline All residents & $0(0-1)^{\mathrm{b}}$ & $0(0-2)^{b}$ & $2(0-5)^{\mathrm{a}}$ & $5(2-9)^{a, b}$ \\
\hline Level $1(\mathrm{n}=15)$ & $1(0-2.5)$ & $0(0-2)$ & $3(0-5)$ & $4(2-11)$ \\
\hline Level $2(n=16)$ & $0(0-1.3)$ & $0.5(0-2.3)$ & $3.5(0-5.75)$ & $8(5-11)$ \\
\hline Level $3(n=18)$ & $0(0-1)$ & $0(0-1.1)$ & $1(0-4.9)$ & $4(2-7.0)$ \\
\hline \multicolumn{5}{|l|}{ Insertion time $(s)$} \\
\hline All residents & $16(11-34)^{\mathrm{b}}$ & $20(11-32)^{\mathrm{b}}$ & $39(19-80)^{\mathrm{a}}$ & $113(44-220)^{\mathrm{a}, \mathrm{b}}$ \\
\hline Level $1(\mathrm{n}=15)$ & $29(13-63)$ & $21(14-51.5)$ & $62(24.5-111.5)$ & $105(44.5-287)$ \\
\hline Level $2(\mathrm{n}=16)$ & $22(14.8-27.5)$ & $20(9.8-27.5)$ & $47.5(20.3-89.3)$ & $137(83.8-195.3)$ \\
\hline Level $3(\mathrm{n}=18)$ & $11(10-26.4)^{\mathrm{c}}$ & $19.5(11.3-32.4)$ & $35.5(18.3-71.1)$ & $78(41-140.7)$ \\
\hline \multicolumn{5}{|l|}{ Needle visibility } \\
\hline All residents & $0.6(0.27-0.75)$ & $0.53(0.19-0.75)$ & $0.22(0.11-0.5)$ & $0.06(0.03-0.13)^{\mathrm{a}, \mathrm{b}}$ \\
\hline Level $1(n=15)$ & $0.32(0.21-0.77)$ & $0.38(0.17-0.80)$ & $0.20(0.08-0.26)$ & $0.04(0.02-0.08)$ \\
\hline Level $2(\mathrm{n}=16)$ & $0.59(0.28-0.75)$ & $0.54(0.34-0.62)$ & $0.24(0.18-0.34)$ & $0.06(0.03-0.10)$ \\
\hline Level $3(n=18)$ & $0.72(0.52-0.76)$ & $0.55(0.21-0.83)$ & $0.23(0.11-0.55)$ & $0.1(0.05-0.14)$ \\
\hline \multicolumn{5}{|c|}{ Number of residents who rated as most difficult view (percentage) } \\
\hline All residents & $1(2 \%)$ & $2(4.1 \%)$ & $9(8.4 \%)$ & $37(75.5 \%)$ \\
\hline Level $1(n=15)$ & $1(6.7 \%)$ & $1(6.7 \%)$ & $3(20 \%)$ & $10(66.7 \%)$ \\
\hline Level $2(n=16)$ & $0(0 \%)$ & $1(6.3 \%)$ & $3(18.8 \%)$ & $12(75 \%)$ \\
\hline Level $3(n=18)$ & $0(0 \%)$ & $0(0 \%)$ & $3(16.7 \%)$ & $15(83.3 \%)$ \\
\hline
\end{tabular}

Table 1. Performance of residents in four cannulation views and self-assessment $(n=49)$. Data were described as Median (IQR) and number (percentage). ${ }^{\mathrm{a}} P<0.05$ compared with the Neutral group; ${ }^{\mathrm{b}} P<0.05$ compared with the $45^{\circ}$ tilt group; ${ }^{c} P<0.05$ compared with Level 1 subgroup. Insertion Time: from skin puncture to aspiration of fluid; Needle visibility: fraction of time the needle tip was visualized during the insertion; Level 1: no experience of ultrasound-guided procedures; Level 2: have performed more than one but less than forty cases of ultrasound-guided procedures; Level 3: have performed forty cases or more.

\begin{tabular}{|c|c|}
\hline & $\mathbf{n}(\%)$ \\
\hline \multicolumn{2}{|l|}{ Gender } \\
\hline Male & $25(51.0)$ \\
\hline Female & $24(49.0)$ \\
\hline \multicolumn{2}{|c|}{ Prior experience of ultrasound usage ${ }^{a}$ (times) } \\
\hline 0 & $15(30.6)$ \\
\hline $1-39$ & $16(32.7)$ \\
\hline$\geq 40$ & $18(36.7)$ \\
\hline
\end{tabular}

Table 2. Demographic variable of participating residents. ${ }^{a}$ Prior experience of ultrasound usage includes ultrasound-guided nerve blocks or central venous catheterization.

significantly, and needle visibility (fraction of time the needle tip was visualized during the insertion) decreased significantly, as compared with the other three groups. This indicates that although $45^{\circ}$ rotation of probe alone will not impede the alignment, it will further increase the difficulty of needle-beam alignment when coexisted with $45^{\circ}$-tilt.

The self-assessment results showed that among the four different views, the $45^{\circ}$-rotation plus $45^{\circ}$-tilt view was rated as the most difficult by $75.5 \%$ residents (Table 1 ). These self-assessment results were consistent with the residents' performance result.

According to prior experiences of ultrasound-guided procedures (Table 2), we divided the residents into three groups including Level 1 (no experience), Level 2 (have performed more than one but less than forty cases) and Level 3 (have performed forty cases or more). A subgroup analysis on the residents' performance found that only in the Neutral view, the Level 3 residents showed less needle redirections $(P=0.011)$ and shorter insertion 
time $(P=0.028)$ when compared with Level 1 residents (Table 1$)$. In other three insertion views, there were no significant differences in the residents' performances between all three subgroups (Table 1).

\section{Discussion}

It is recommended to utilize an in-plane technique for ultrasound-guided procedures because the entire needle and the depth of the needle tip can be visualized on the ultrasound image $e^{2,7}$. However ultrasound might give the inexperienced operators a false sense of security and mislead them to neglect traditionally taught principles with regard to needle direction. Blind advancement of the needle can lead to accidental damage of vital structures like artery or pleura ${ }^{8,9}$. It is important to identify the potential factors that may complicate the alignment of needle with ultrasound beam. These factors can be used to improve the clinical relevance of simulation training and quantitatively assess the difficulty of ultrasound-guided procedures.

Tilting and rotating are often used to optimize the visualization of the targeted nerves and bring the "disappeared" needle tip back ${ }^{10,11}$. In this phantom study, we found that the tilted probe will increase the difficulty of the needle-beam alignment. When the operators were faced with both rotation and tilt angles, the alignment is further complicated. The possible reason may be that the operators were less willing to tilt their head or body to keep the line of sight parallel to the ultrasonic beam plane. It is recommended that the operator should have the insertion site, the needle, and the ultrasound screen in the line of sight during needle insertion ${ }^{2,10}$. Our study indicates that if a probe tilt angle exists, the operator shall tilt their body or head to keep the line of sight parallel to the ultrasonic beam plane, in order to facilitate the needle-beam alignment.

Although in our study the $45^{\circ}$ rotation of probe did not lead to a decreased performance, another phantom study found that needle advancement along the visual axis resulted in improved needle imaging and a shorter time to targeting when compared with that across the visual axis $\left(90^{\circ} \text { rotation of probe }\right)^{12}$. It is advisable for the operators to move their standing position to eliminate the rotation angle of probe.

Previous experiences of operator are presumed to be helpful to needle-beam alignment. However in our study, the level 3 residents who had performed more than 40 cases of ultrasound-guided procedures only showed better performance in the Neutral view while not did in $45^{\circ}$-rotation and/or $45^{\circ}$-tilt views. However, the sample size of subgroup was not large enough to make a reliable conclusion. The difference between phantom research and clinical practice may also complicate the extension of these results which warrants a well-designed clinical research.

In conclusion, our study found that both $45^{\circ}$-tilt and $45^{\circ}$-rotation plus $45^{\circ}$-tilt of probe would increase the difficulty of needle-beam alignment during in-plane procedures. Operators with experiences of ultrasoundguided procedures might also be affected by these probe manipulation angles. It is advisable for the operators to move their standing position and tilting their body or head to keep the line of sight parallel to the ultrasonic beam plane, in order to facilitate the needle-beam alignment.

Received: 8 September 2020; Accepted: 6 January 2021

Published online: 15 January 2021

\section{References}

1. Vogel, J. A. et al. Is long-axis view superior to short-axis view in ultrasound-guided central venous catheterization?. Crit. Care Med. 43, 832-839 (2015).

2. Lamperti, M. et al. International evidence-based recommendations on ultrasound-guided vascular access. Intens. Care Med. 38 , 1105-1117 (2012).

3. Neal, J. M. Ultrasound-guided regional anesthesia and patient safety: update of an evidence-based analysis. Reg. Anesth. Pain Med. 41, 195-204 (2016).

4. Marhofer, P. \& Fritsch, G. Safe performance of peripheral regional anaesthesia: the significance of ultrasound guidance. Anaesthesia 72, 431-434 (2017).

5. van Geffen, G. J. et al. Correlation between ultrasound imaging, cross-sectional anatomy, and histology of the brachial plexus: a review. Reg. Anesth. Pain Med. 34, 490-497 (2009).

6. Muhly, W. T. \& Orebaugh, S. L. Sonoanatomy of the vasculature at the supraclavicular and interscalene regions relevant for brachial plexus block. Acta Anaesthesiol. Scand. 55, 1247-1253 (2011).

7. Taylor, A. \& Grant, C. R. K. Complications of regional anaesthesia. Anaesth. Intens. Care Med. 20, 210-214 (2019).

8. Saugel, B., Scheeren, T. W. L. \& Teboul, J. L. Ultrasound-guided central venous catheter placement: a structured review and recommendations for clinical practice. Crit. Care 21, 225 (2017).

9. Maecken, T., Heite, L., Wolf, B., Zahn, P. K. \& Litz, R. J. Ultrasound-guided catheterisation of the subclavian vein: freehand vs needle-guided technique. Anaesthesia. 70, 1242-1249 (2015).

10. Chin, K. J., Perlas, A., Chan, V. W. \& Brull, R. Needle visualization in ultrasound-guided regional anesthesia: challenges and solutions. Reg. Anesth. Pain Med. 33, 532-544 (2008).

11. Phelan, M. \& Hagerty, D. The oblique view: an alternative approach for ultrasound-guided central line placement. J. Emerg. Med. 37, 403-408 (2009).

12. Wilson, J. M., Germain, G., Vaghadia, H., Tang, R. \& Sawka, A. In-plane ultrasound-guided needle insertion ALONG or ACROSS the visual axis hand positions. Br. J. Anaesth. 113, 717-718 (2014).

\section{Acknowledgements}

The authors would like to thank all the residents, especially Dr. Jing Chen, Jun Zhang, Ruibo Yang and Lijun Wang, for participating in the study and Ruodan Gan for help with Fig. 1a-d illustration.

\section{Author contributions}

Q.M., H.Y. and L.C. contributed to study design and the drafting of the manuscript; H.H. and Y.L. contributed to statistical revision, reading and checking of the manuscript; Y.H. and Z.W. contributed to data analysis, and reading and checking of the manuscript; M.G., Q.M., H.H. and Y.L. contributed to resident recruitment and data collection. All authors reviewed the paper and agreed with final version of the manuscript. 


\section{Funding}

This research was supported by the National Natural Science Foundation of China (Grant No. 81701116) and Natural Science Foundation of Chongqing (Grant No. cstc2019jcyj-msxmX0018).

\section{Competing interests}

The authors declare no competing interests.

\section{Additional information}

Correspondence and requests for materials should be addressed to H.Y. or L.C.

Reprints and permissions information is available at www.nature.com/reprints.

Publisher's note Springer Nature remains neutral with regard to jurisdictional claims in published maps and institutional affiliations.

(c) (i) Open Access This article is licensed under a Creative Commons Attribution 4.0 International License, which permits use, sharing, adaptation, distribution and reproduction in any medium or format, as long as you give appropriate credit to the original author(s) and the source, provide a link to the Creative Commons licence, and indicate if changes were made. The images or other third party material in this article are included in the article's Creative Commons licence, unless indicated otherwise in a credit line to the material. If material is not included in the article's Creative Commons licence and your intended use is not permitted by statutory regulation or exceeds the permitted use, you will need to obtain permission directly from the copyright holder. To view a copy of this licence, visit http://creativecommons.org/licenses/by/4.0/.

(C) The Author(s) 2021 